\title{
Jean de Montaigu y el Castillo de Marcoussis: Un señorío en construcción en torno al año 1400
}

\section{Nuria Laorga Fernández ${ }^{a}$}

${ }^{a}$ Doctoranda Paris I Panthéon Sorbonne, 14 rue du Général Mangin, 92600 Asnieres sur Seine, Francia, nuria.laorga@gmail.com.

\begin{abstract}
Resumen
La locura del rey Charles VI; el reino de Francia prometido al rey de Inglaterra por medio del tratado de Troyes; la trágica muerte en la hoguera de Juana de Arco; la aparición del personaje de la muerte en el arte... Varios hechos que nos muestran hasta qué punto los siglos XIV y XV forman parte de una época crucial y problemática. Época en la que no era nada fácil conservar un estatus social, generalmente alcanzado gracias a las ayudas de los príncipes y a los enormes gastos en la construcción de imponentes edificios que aseguraban la grandeza de estos personajes.

Jean de Montaigu fue más allá de las guerras externas e internas y luchó para lograr sus objetivos, aunque esto le costó la vida. Al sur de Paris edificó su señorío en el que incluyó la iglesia parroquial, un convento y su magnifico castillo.
\end{abstract}

Palabras clave: arquitectura, medieval, Francia, castillo, convento, iglesia, guerras.

\begin{abstract}
King Charles VI madness; the kingdom of France promised to the King of England through the Traty of Troyes; the tragic death at the stake of Joan of Arc; the appear of the carácter of death in arts... Several facts that show us how far the 14th and 15th centuries make part of a crucial and problematic era. In that time it was not easy to maintain a social status, generally achieved thanks to the help of princes and the enormous expenses in the construction of imposing buildings that ensured the greatness of those characters.

Jean de Montaigu went beyond the external and internal wars and struggled to achieve his goals, even if that took his own life. In the south of Paris he built his manor in which he included the parish church, a convent and his magnificent castle.
\end{abstract}

Keywords: architecture, medieval, France, castle, convent, church, wars. 


\section{Introducción}

Una alta torre y un imponente foso de principios del siglo XV son de los pocos restos que nos quedan del señorío de Marcoussis, muestra del poder y elevado estatus social de su fundador, Jean de Montaigu (Fig. 1).

Este estudio no pretende realizar una descripción estilística de los monumentos que en su día formaron este señorío, sino abordar de manera resumida la importancia de mantener un estatus social en torno al año 1400 a través de la creación de edificios de poder. Para ello haremos un recorrido por la vida de Jean de Montaigu, su increíble ascenso y su trágica muerte en la corte del rey Charles VI, así como la importancia de los monumentos que formaron su ansiado señorío.

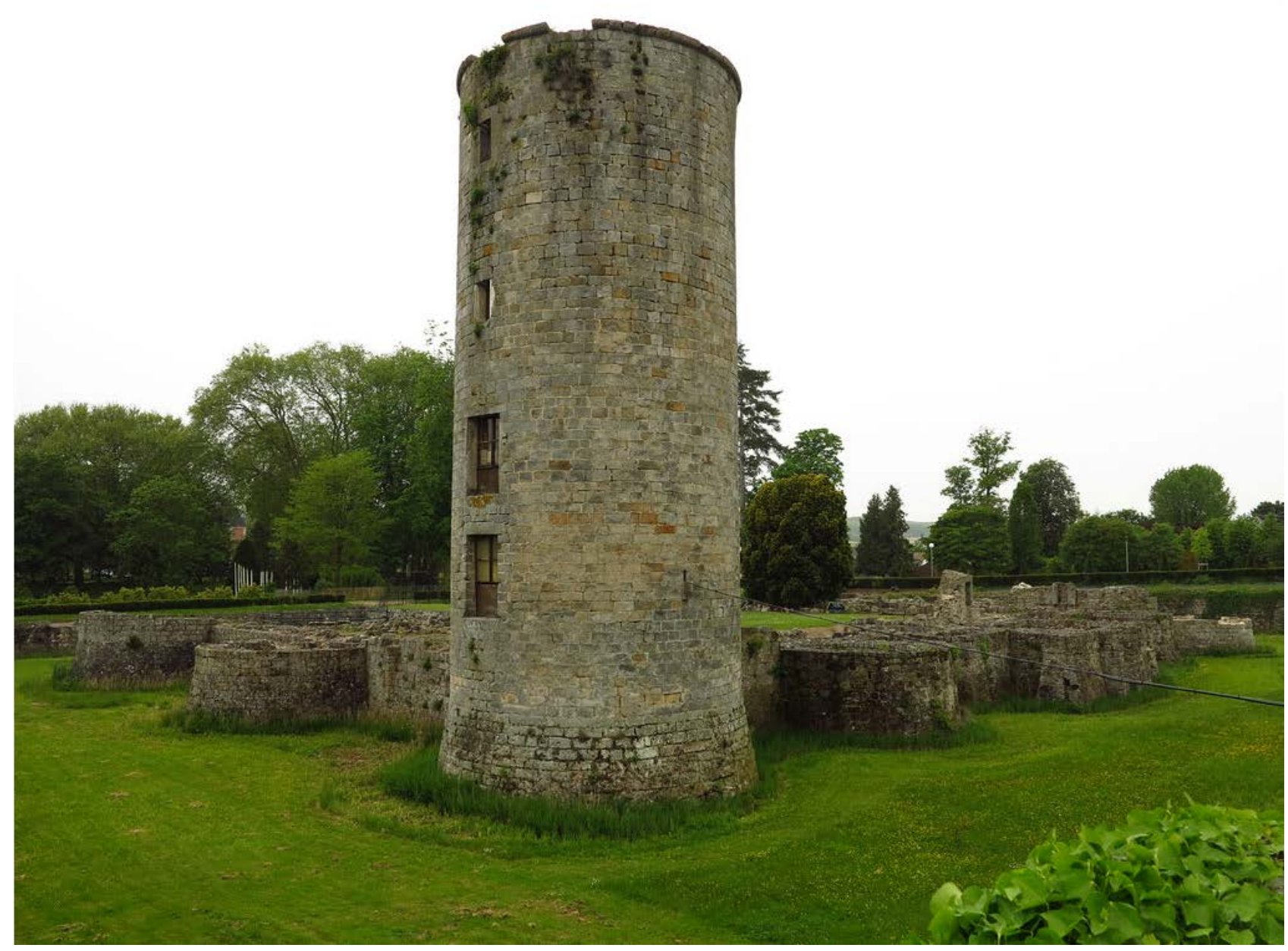

Fuente: Ackteon (2012)

Fig. 1 Torre del castillo de Marcoussis

Jean de Montaigu es la tercera generación de tesoreros del rey. Su padre Gerard de Montaigu y su madre Biette de Cassinel, tenían una buena relación con el rey Charles V, lo que le permitió estar en el entorno de la corte real desde muy temprana edad (Plagnieux, 2006).

La vida de Jean de Montaigu se caracteriza por una gran ambición de ascender a lo más alto en la escala social y aumentar su fortuna, a imagen de la de sus padres. Esto se traduce en la compra de numerosos bienes y territorios, que se añaden a los muchos que ya había adquirido su padre a lo largo de los años. Pero el verdadero apogeo de su evolución social se ve reflejado en la construcción de su señorío, de su castillo.

Empieza siendo secretario del rey Charles V, pero será bajo el reinado de Charles VI cuando su ascenso social alcance la cima. Su matrimonio con Jacqueline de La Grange, sobrina del cardenal de Amiens, primer ministro de Charles V, supone una alianza matrimonial que muestra el poder adquirido por Jean de Montaigu. Poder, que no hará sino crecer 
hasta el resto de sus días. Podemos decir que este enlace matrimonial es el comienzo de su señorío, ya que fue su tío Ferry de Cassinel quien le dona las tierras de Marcoussis como regalo de bodas.

\section{Un agitado contexto histórico}

En 1380 muere Charles V y accede al trono su hijo con tan solo doce años. Siendo tan joven el nuevo rey, se establece la tutela de sus tíos: Louis duque de Anjou, Jean duque de Berry y Philippe le Hardi duque de Borgoña por parte de su padre, Louis II el bueno, duque de Bourbon por parte de su madre.

Desde ese momento las rivalidades entre los príncipes regentes están presentes en la corte. El duque de Anjou quiere reinar él solo, alegando su mayoría de edad. Los otros príncipes no están de acuerdo en dejarle la tutela al duque de Anjou y proponen crear un consejo en el que todos fueran miembros. Sin embargo, la tensión es tan fuerte que acaban por aceptar la regencia del duque de Anjou hasta el día del sacro de Charles VI. Día que vería emanciparse al rey y las decisiones del reino pasarían a ser tomadas por el consejo (Bainville, 2014).

Los duques estaban contentos con esta nueva situación, aunque en realidad no era nada buena para el reino. Cada uno de ellos miraba por sus propios intereses y ambiciones, convirtiendo el gobierno en un completo desorden, sobre todo en el ámbito de las finanzas.

Frente a esta situación, los consejeros de Charles VI conocidos como los Marmousets, incitan al rey a disolver este consejo poco propicio al reino. Una vez apartados del poder (aunque no por mucho tiempo), serán los Marmousets los que se encarguen de la administración del reino. Entre los que formaban este grupo tan cercano al monarca se encontraba Jean de Montaigu.

Siendo ya secretario del rey, Jean de Montaigu mostrará en diferentes momentos su talento en el ámbito económico, consiguiendo así el nombramiento por parte del rey de Subintendente de las finanzas reales. Siempre junto a Charles VI, se vuelve indispensable tanto para él como para su hermano el duque de Orleans. Pero su ambición de poder iba acompañada de una gran prudencia, por lo que no dejaba de lado a los tíos del monarca, el duque de Berry y el duque de Borgoña, interesados todavía en la toma del poder (Merlet, 1852).

Ambos duques conseguirán su objetivo y retomarán el poder durante un breve periodo de tiempo, tras la catástrofe del bosque de Le Mans. En efecto, en medio del bosque de Le Mans, se produce la primera crisis de demencia de Charles VI, quien matará a varios de sus hombres. Cuando el duque de Berry y el de Borgoña retoman la tutela del reino, ordenan a Jean de Montaigu que se aparte del rey, instalándose en Aviñon (Schnerb, 2009).

Las crisis de salud del rey se hacen cada vez más frecuentes y la relación entre los tíos del rey y su hermano cada vez más tensa. En 1404 este último acaba siendo nombrado teniente del reino, el tiempo que fuera necesario para que el rey se restableciera.

Ese mismo año murió el duque de Borgoña, a quien sucedió su hijo conocido como Juan sin Miedo. El nuevo duque no tenía la misma influencia que su padre tenía en la corte y para con la reina, sin embargo, su ambición por el poder era incluso mayor. Para alcanzar sus fines decide comenzar por deshacerse del hermano del rey. Así en 1407 envía sus soldados a matar al duque de Orleans en las calles de Paris.

Mientras tanto, Jean de Montaigu se creía todavía bajo la gracia de los reyes y el duque de Berry, y no le preocupaba que sus allegados le dijesen que el duque de Borgoña había igualmente jurado su muerte.

Aprovechando el estado de salud del rey, bajo pretexto de una revisión del estado de las finanzas, Juan sin Miedo consigue encarcelar a Jean de Montaigu, quien tras ser torturado será condenado a muerte cortándole la cabeza en 1409 (Merlet, 1852).

La historia del ascenso social de Jean de Montaigu y todas las peripecias que llevaron a su condena aún estando bajo la protección del rey, son los síntomas de una época de guerra externa, civil y conflictos entre los príncipes. Un conjunto de dificultades que hoy conocemos como la Guerra de los 100 años, marcada además por hambrunas, pestes y cisma en el seno de la iglesia. 


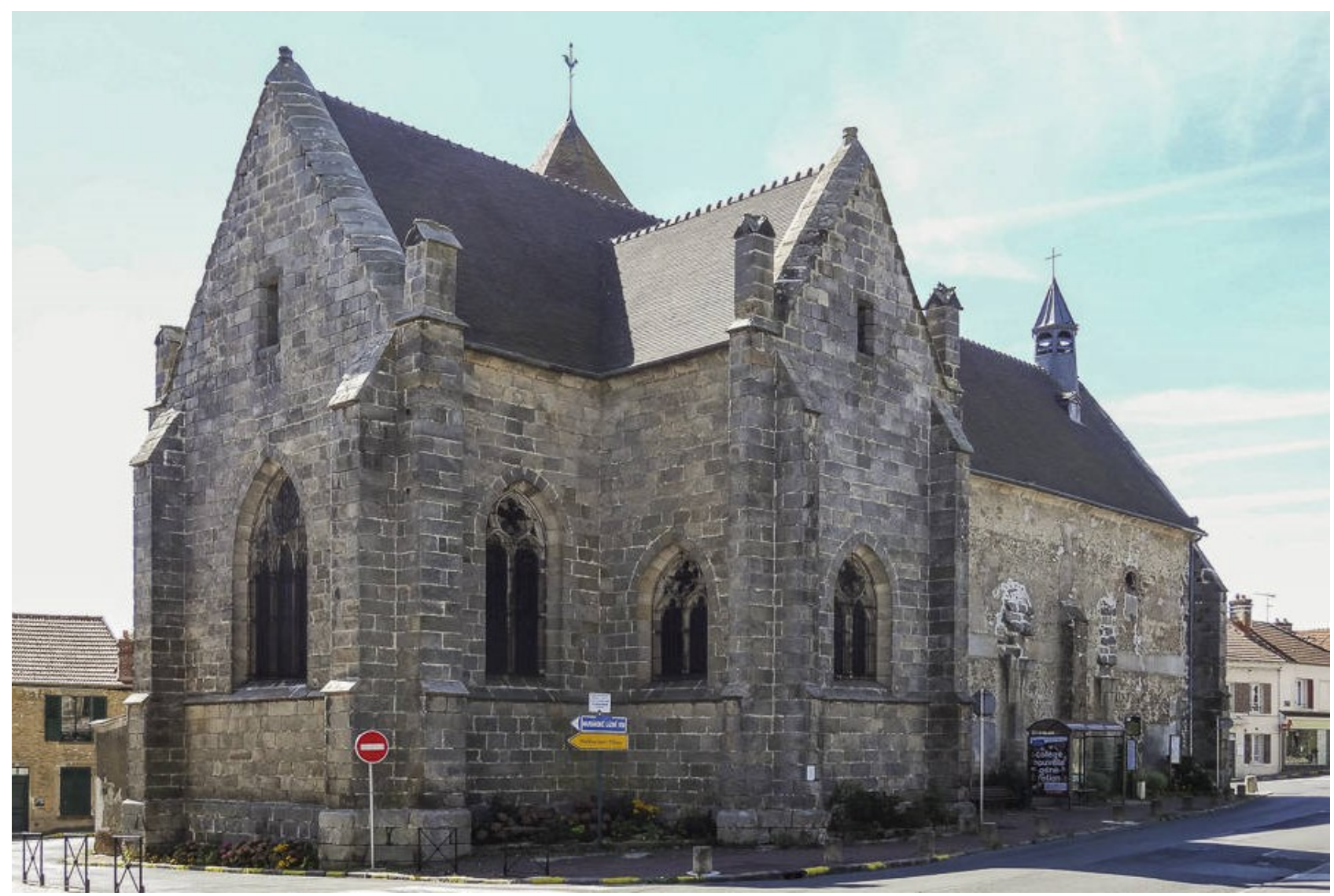

Fuente: Evasions Culturelles (2011)

Fig. 2 Iglesia de Marcoussis

\section{El señorío de Marcoussis}

Durante toda su vida, Jean de Montaigu vivió en diferentes hôtels particuliers ${ }^{l}$ en la ciudad de Paris. Entre otros conocía el Louvre y la Bastilla, de la que también fue capitán. Era perfectamente consciente de la importancia que conferían las realizaciones arquitectónicas de los príncipes, signos de poder y rango social elevado. Sobre todo, en lo que concierne a los duques de Berry y Orleans, quienes tenían sus propios arquitectos y ordenaron numerosas construcciones.

En 1388, como regalo de boda por parte de su tío el obispo de Auxerre, Ferry de Cassinel, adquiere las tierras de Marcoussis, hecho que le permite aumentar su fortuna y reforzar su posición social y señorial junto al rey. Jean de Montaigu decide hacer de su señorío el emblema de su nuevo poder y comienza una obra arquitectónica de gran envergadura. Primero la construcción de un castillo, luego el coro de la iglesia parroquial y por último un convento para los Celestinos. La idea de construir este último surge tras la primera crisis de locura del rey (Merlet, 1852).

Los tres edificios se construyeron entre 1402 y 1408, un espacio de tiempo bastante corto (Germain, 1973). La principal razón del retraso o paro total en las obras medievales era la falta de dinero. Juan de Montaigu, al consagrar una gran parte de su inmensa fortuna a la realización de estos trabajos, no tuvo este tipo de problemas.

Además de sus proyectos arquitectónicos, va a crear estanques y jardines para hacer de su señorío un lugar bonito de descanso.

Según un monje celestino del siglo XVII, se contrató un número enorme de obreros y había siete forjas que trabajaban sin descanso día y noche para preparar las herramientas (Langre, 1694).

${ }^{1}$ Mansion o palacete edificado en ciudad. 


\subsection{La Iglesia parroquial}

Iglesia de tipo rural, sin nártex, el acceso da directamente sobre la nave. Cuando Juan de Montaigu llega a Marcoussis la encontró en ruinas y decidió reconstruirla.

En el siglo XII, cuando se edificó la iglesia, se empleó un aparejo irregular formado por pequeñas piedras y otros materiales heterogéneos. La piedra que utiliza Juan de Montaigu es la misma que en el resto de sus edificios, a saber, un aparejo regular constituido por bloques de arenisca gris.

Empieza rehaciendo el coro, al que añade una capilla señorial y su intención era continuar con el resto de la nave. Su proyecto se ve frenado por el prior de San Wandrile, patrón de la Iglesia, por miedo a un cambio en el patronato de la misma. Hay que tener en cuenta que en la Edad Media, era el obispo quien decidía el patrón de las iglesias junto con los fundadores o las personas que mandan su consagración (Germain, 1973).

Obligado a terminar su obra, se ve en la obligación de alzar los muros de la nave para que estén a la misma altura que el coro y poder realizar la cubierta. Esta elevación se distingue muy bien hoy en día por la diferencia de color del mortero empleado. También podemos observar una serie de contrafuertes más altos para sostener el peso de la nave (Fig. 2).

En el interior, manda plasmar sus armas en las claves de las bóvedas y en los arcos de la capilla.

\subsection{El Convento de los Celestinos}

Como ya hemos mencionado con anterioridad, tras el crucial día en el bosque de Le Mans, advertido de no acercarse al rey y con el fin de protegerse de los tíos del monarca, Montaigu se instala en Aviñón. Aquí decide construir un convento consagrado a la Santísima Trinidad, para invocar a Dios por la salud del rey (Germain, 1973).

Una vez en Marcoussis comienza la construcción del convento, que estaba situado a un kilómetro aproximadamente del castillo, y lo cede a la orden de los Celestinos. Parece ser que su idea era construir una pasarela que uniera ambos edificios con el fin de facilitar el acceso cuando quisiera asistir al oficio.

Hoy en día quedan solamente unos pocos vestigios de este conjunto monástico. Unos cuantos lienzos de muros y las bodegas (Fig. 3), conservadas en un excelente estado.

\subsection{El castillo de Marcoussis}

El castillo es seguramente la parte más interesante del señorío al ser el resultado final de una larga evolución de la arquitectura de castillos en la Edad Media.

Con el objetivo de evocar de manera muy resumida esta transformación de los castillos en Francia vamos a tomar como punto de referencia la Edad Media Clásica, en concreto el reinado del rey Felipe II Augusto, entre 1180 y 1223. Hasta entonces, en los siglos X-XII los castillos se limitaban a una elevación del terreno coronada por una torre del homenaje rodeada por una muralla de madera. Las dos funciones básicas del edificio estaban bien separadas. Por un lado la función de residencia, en la torre del homenaje, y la función defensiva, la muralla.

Felipe Augusto introduce un tipo de castillo que se presenta cada vez más en un solo bloque. Un bloque que es al mismo tiempo residencial y defensivo. En un primer momento la torre del homenaje se incluye en la muralla y poco a poco se van añadiendo en las esquinas torres circulares de base compacta destinadas a la defensa. La muralla va a desaparecer para dar lugar a un foso que rodea el conjunto Philippien (de Felipe Augusto) (Mesqui, 1991).

La Bastilla y el castillo de Vincennes, en los cuales se inspira Jean de Montaigu para su castillo en Marcoussis, aportan un nuevo reparto de roles en las torres. Entre los niveles de defensa habrá espacios residenciales. Las bases ya no son compactas, sino que introducen un sistema defensivo de tiro raso completado por los pisos superiores de la torre y las terrazas.

El castillo de Marcoussis empieza a construirse en 1402 sobre los restos de una antigua fortaleza. Su planta cuadrangular está formada por cuatro cuerpos principales que albergaban habitaciones, cocinas, hornos, despensas... En las cuatro esquinas se levantaban cuatro torres con habitaciones, chimeneas y letrinas, a imagen de las torres del modelo Philippien. Todo rodeado por un foso. 


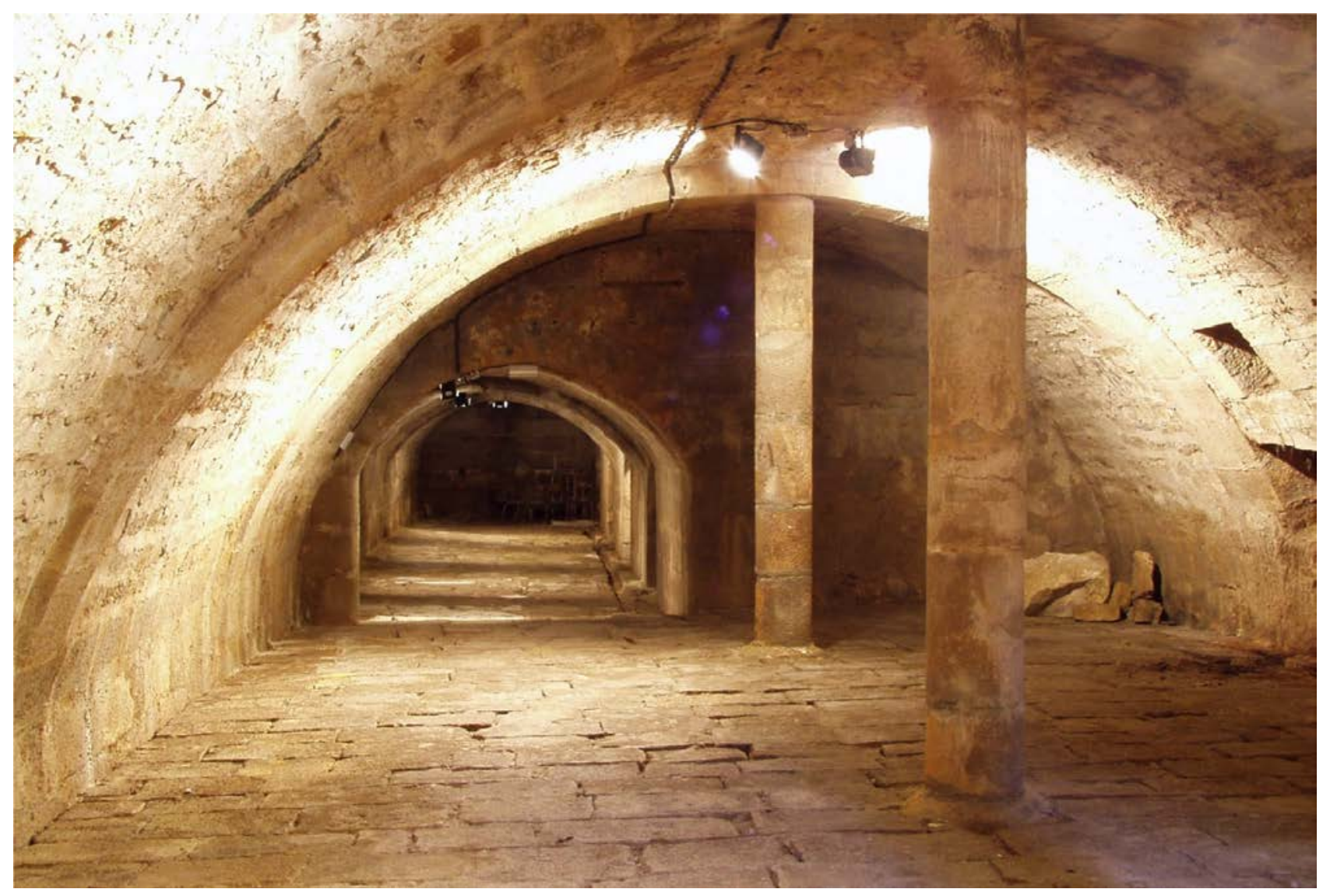

Fuente: Laorga Fernández, N. (2016)

Fig. 3 Bodegas del convento

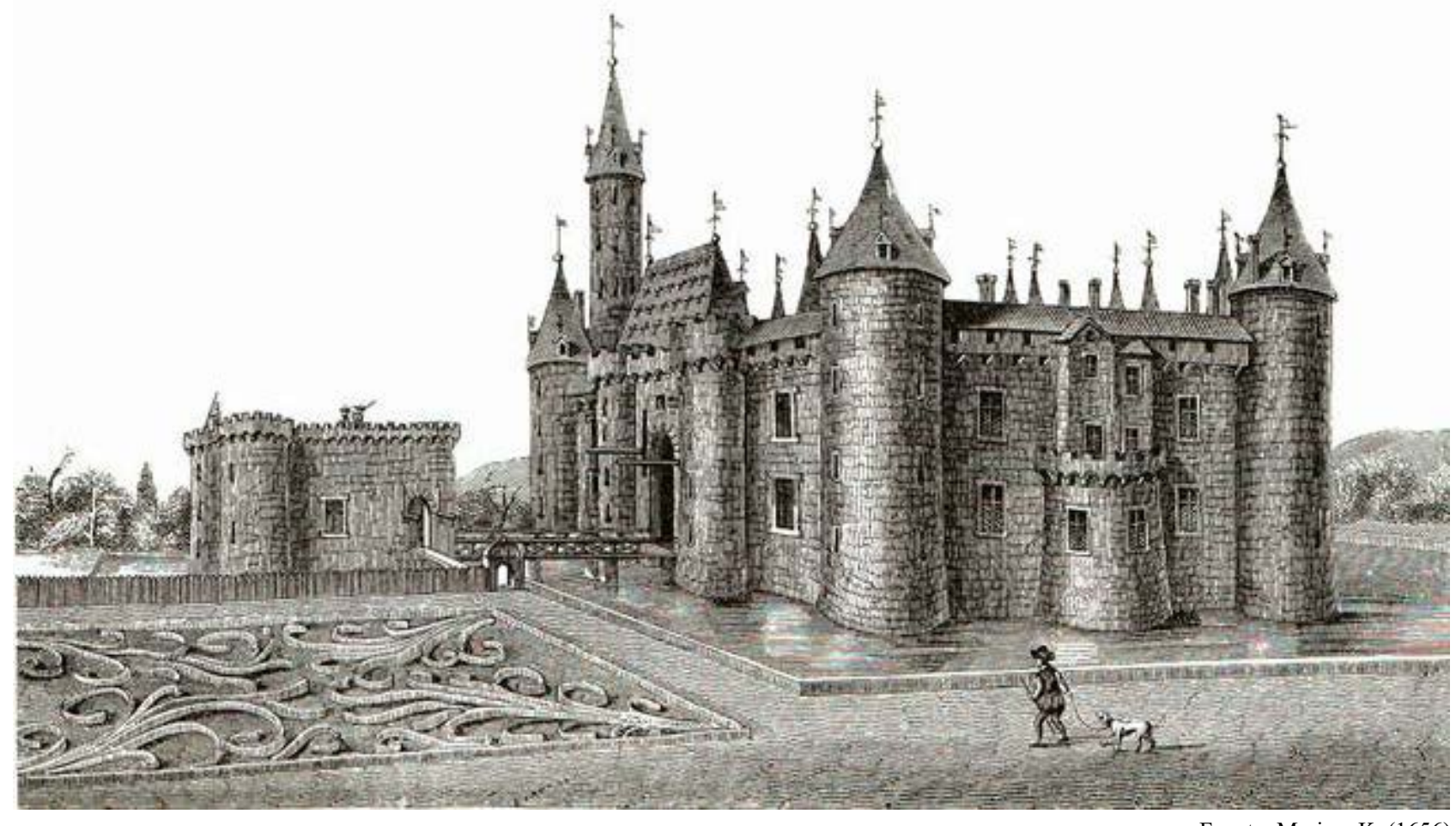

Fuente: Merian, K. (1656)

Fig. 4 Vista del castillo y la barbacana 
El acceso se hacía por el lado sur, a través de un puente levadizo protegido por una gran torre del homenaje flanqueada por torreones. Al otro lado del puente hay otro edificio cuadrangular, una barbacana construida seguramente más tarde que el castillo, finales del siglo XV principios del XVI. Tras ser destruido en la revolución francesa, la única torre en pie pasó a ser una capilla hacia el 1827 (Fig. 4).

Para Montaigu su señorío era la muestra de su poder y posición social, pero sus enemigos veían más bien un monumento a su orgullo. Monumento que como el resto de las construcciones de los príncipes, se habría construido gracias a las finanzas del reino. Este será un argumento más utilizado cuando le condenen a muerte (Moranvillé, 1890).

De este imponente edificio símbolo de poder casi desaparecido en nuestros días, solo nos quedan las fundaciones, el foso y una hermosa torre. En 1805 fue destruido por el marqués de Puiségur para evitar que lo convirtiesen en prisión del estado y el lugar cayó en el olvido, arropado por la vegetación. Un espeso manto vegetal cubriendo un verdadero tesoro arqueológico.

\section{Conclusión}

Llevar una vida de lujo no es algo que pudiera permitirse cualquier persona en la Edad Media. Si Jean de Montaigu lo consigue, es por una parte gracias a su inteligencia, y por otra al afecto que los reyes y principes sienten por él. Gracias a su buen trabajo e impliación en las tareas reales y a los numerosos regalos obtenidos, consiguió una enorme fortuna que invertió en su obra maestra: su señorío en Marcoussis. No obstante, sus ambiciones quizás eran algo extremas para los tiempos convulsos en los que vivía. Pese a que los parisinos empezaban a mostrarse descontentos con los elevados gastos de la corte, Jean de Montaigu seguía aumentando y enriqueciendo su patrimonio. Sus enemigos no tardaron entonces en acusarlo de traición y en 1409 le cortaron la cabeza.

Una vez conseguido el afecto de los personajes más importantes del reino y un gran poder en la sociedad, solo queda plasmarlo de manera física. Teniendo los recursos necesarios, Jean de Montaigu no se contentó con edificar un simple señorío. Sus deseos iban más allá y quiso realizarlo a imagen de las construcciones de los príncipes. Un ejemplo de ello es la presencia en su castillo de dos capillas superpuestas, algo que vemos en construcciones de la realeza, como la Sainte Chapelle de París.

Los restos arqueológicos que han llegado hasta nosotros y los textos, casi todos tardíos, nos ayudan a imaginar la importancia de esas construcciones. Como dice Perron de Langre (1694), esta arquitectura de orden antiguo y gótico nos muestra los vastos deseos que podía tener Jean de Montaigu, ya que su castillo, construido en tan poco tiempo, tiene todos los signos de una fortaleza de defensa impenetrable.

\section{Referencias}

Bainville, J. (2014). Histoire de France. Paris: Perrin.

Carpentier, J., y Lebrun, F. (Dir.). (2000). Histoire de France. France: Editions du Seuil.

Germain, H. (1973). Marcoussis: département de l'Essone, canton de Montlhéry, le réveil de son histoire et monographie. BoulogneBillancourt.

Langre, P. (1694). L'anastase de Marcoussis ou recherches curieuses de son origin, progrès et agrandissements. Paris.

Merlet, L. (1852). Biographie de Jean de Montaigu, grand maître de France (1350-1409). Bibliothèque de l'Eole des Chartes, 13, 248-284.

Mesqui, J. (1991). Châteaux et enceintes de la France Médiévale: de la défense à la résidence. Paris: Picard.

Moranvillé, H. (1890). Le songe véritable. Pamphlet politique d'un parisien du XVe siècle. Mémoires de la Societé de l'Histoire de Paris et de l'Ile de France, XVII, 299-300.

Plagnieux, P. (2006). Jean de Montaigu ou la résistible ascension d'un parvenu à la lumière des arts. En E. Taburet-Delahaye (Ed.), La création artistique en France autour de 1400 (pp. 103-118). Paris: Rencontres de l'Ecole du Louvre.

Schnerb, B. (2009). Armagnacs et Bourgignons. La maudite guerre 1407-1435. Paris: Perin. 